\title{
Solute Concentration in the Kidney- II. Input-Output Studies on a Central Core Model*
}

\author{
DAVID FOSTER, JOHN A. JACQUEZ AND EUGENE DANIELS \\ The Department of Physiology, The Medical School, \\ The University of Michigan, Ann Arbor, Michigan 48109
}

\begin{abstract}
Input-output studies have been carried out on a central core model of the renal medulla of the rabbit. The results emphasize the importance of having a nontransported solute such as urea. Even though only salt was actively transported, in the thick AHL. urea had to be present for a concentration gradient to be built up. Inflow into the DHL was shown to be a major determinant of the concentration process. The concentration increased with inflow into DHL up to a maximum value; with further increase in inflow into DHL, the concentration obtained decreased. Extrapolating from these studies and what is known of the role of flow in vasa rectae we argue that independent control of afferent and efferent arteriolar resistance of juxtamedullary nephrons could play a major role in control of the concentration of urine.
\end{abstract}

\section{INTRODUCTION}

In the previous paper [1], we derived the equations describing two concentrating engines the geometry and operation of which were motivated by our knowledge of the structure and function of the medulla. For the second of these, the central core model, the experimental data necessary to estimate the parameter and input values for the model are available in the literature. Moreover, the numerical techniques necessary to solve the equations are also available.

As we have already seen, the central core model is one of the many possible limit cases of the full model [1]. It is of particular interest, however, because it markedly simplifies the model and because its behavior in response to changes in the parameter and input values provides us with insights into the behavior of more general models and even of the medulla itself.

*This work was supported in part by grants AM 16635 and GM 00110 from the National Institutes of Health, DHEW.

MATIIEMATICAL BIOSCIENCES 32, 337-360 (1976)

(c) American Elsevier Publishing Company, Inc., 1976 
In this paper, we present the results of simulations using the central core model. The data which we will use to obtain the parameter and input values are those which have been obtained on rabbit kidneys. Our first simulations show that with these parameter and input values, a concentration gradient is established in the central core, and a urine flow rate and concentration are generated that lie well within the bounds for those values determined experimentally. We will show that the changes in these values in the presence and absence of ADH are what we would expect. We conclude with a presentation and discussion of the results of a series of input-output studies which are designed to show how the model behaves when subjected to changes in specific input values.

\section{THE CENTRAL CORE MODEL}

A schematic of the central core model is given in Fig. 1. For convenience, the notation for the parameters, inputs, and outputs are indicated on the figure; Table 1 contains a listing of them together with their units and what they represent.

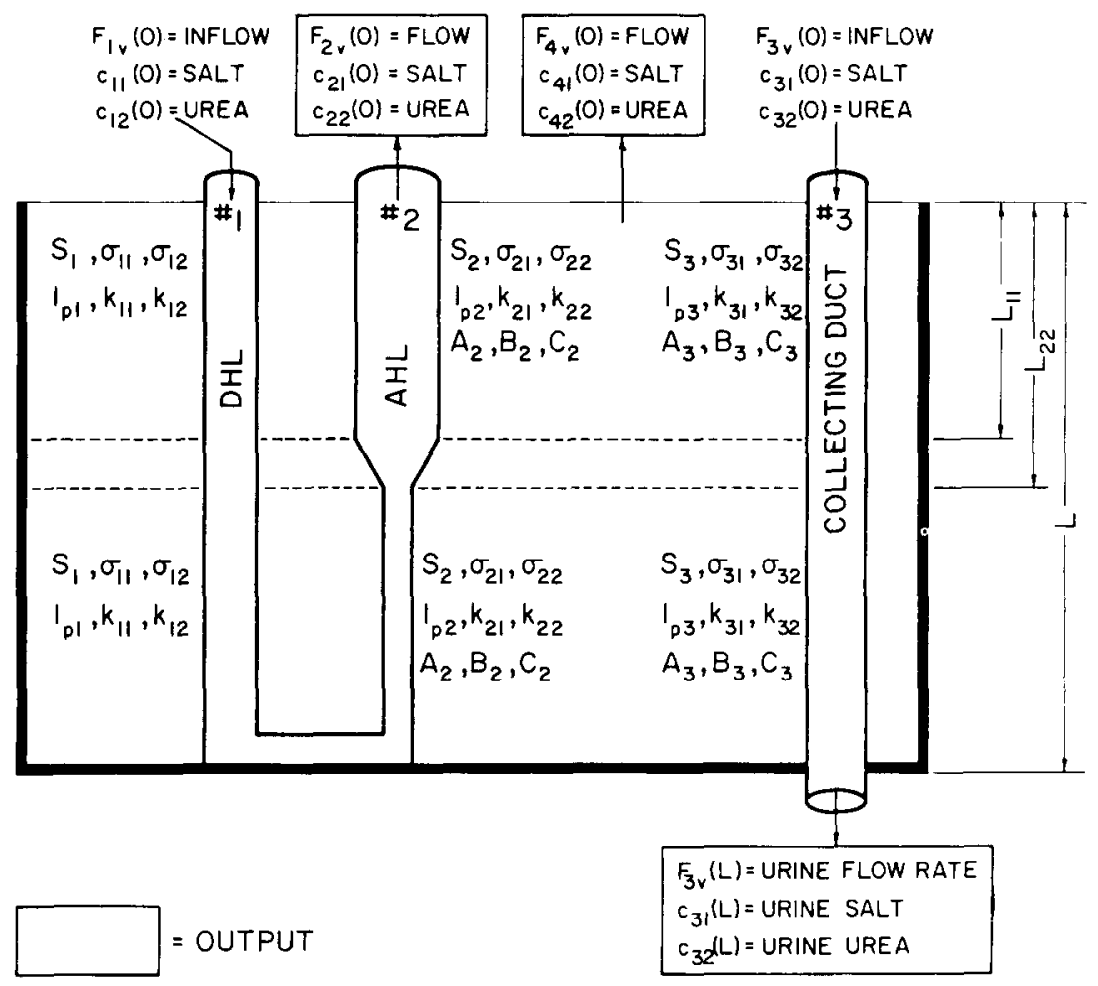

FIG. 1. Schematic diagram of central core model. 
TABLE 1

Description of Symbols Used in the Central Core Model

\begin{tabular}{|c|c|c|}
\hline Symbol & Units & Description \\
\hline$L$ & $\mathrm{~cm}$ & Total width of medulla. \\
\hline$L_{11}$ & $\mathrm{~cm}$ & $\begin{array}{l}\text { Beginning of linear transition from outer } \\
\text { to inner medulla. }\end{array}$ \\
\hline$L_{22}$ & $\mathrm{~cm}$ & $\begin{array}{l}\text { End of linear transition from outer to } \\
\text { inner medulla. }\end{array}$ \\
\hline$x$ & $\mathrm{~cm}$ & $\begin{array}{l}\text { Independent variable. Distance into } \\
\text { medulla } \\
\text { border. }\end{array}$ \\
\hline$k$ & & Solutes: $k=1$ is salt; $k=2$ is urea. \\
\hline$i$ & & $\begin{array}{l}\text { Tube number: } i=1 \text { is DHL; } i=2 \text { is } \\
\text { AHL: } i=3 \text { is } \mathrm{CD} ; i=4 \text { is Central Core. }\end{array}$ \\
\hline$S_{i}$ & $\mathrm{~cm}^{2} / \mathrm{cm}$ & Surface area per unit length of tube $i$. \\
\hline$F_{i v}$ & $\mathrm{ml} / \mathrm{min}$ & Volume flow rate in tube $i$ \\
\hline$F_{i k}$ & $\mathrm{mmoles} / \mathrm{min}$ & Flow rate of solute $k$ in tube $i$ \\
\hline$C_{i k}$ & mmoles $/ \mathrm{cm}^{2}$ & Concentration of solute $k$ in tube $i$. \\
\hline$J_{i v}$ & $\mathrm{~cm}^{3} / \mathrm{cm}^{2} \cdot \min$ & $\begin{array}{l}\text { Volume flux across wall of tube } i \text {, Posi- } \\
\text { tive outwards. }\end{array}$ \\
\hline$J_{i k}$ & $\mathrm{mmoles} / \mathrm{cm}^{2} \cdot \min$ & Flux of solute $k$ across wall of tube $i$. \\
\hline$L_{p i}$ & $\mathrm{~cm}^{3} /$ dyne $\cdot \min$ & $\begin{array}{l}\text { Filtration coefficient for the wall of tube } \\
i \text {. }\end{array}$ \\
\hline$l_{p i}$ & & $\begin{array}{l}\mathrm{RTL}_{p i}, \quad T=37^{\circ} \quad \text { C. } \quad\left(\mathrm{cm}^{3} / \mathrm{cm}^{2} .\right. \\
\min )\left(\mathrm{cm}^{3} / \mathrm{mmole}\right) .\end{array}$ \\
\hline$\sigma_{i k}$ & & $\begin{array}{l}\text { Reflection coefficient of solute } k \text { in tube } \\
i \text {. }\end{array}$ \\
\hline$\omega_{i k}$ & mmoles/dyne $\cdot$ min & $\begin{array}{l}\text { Permeability coefficient of solute } k \text { across } \\
\text { the wall of tube } i \text {. }\end{array}$ \\
\hline$k_{i k}$ & $\mathrm{~cm} / \mathrm{min}$ & $\mathrm{RT} \omega_{i k}, T=3^{\circ} \mathrm{C}$ \\
\hline$A_{i}$ & & Parameter describing the pump in tube $i$. \\
\hline$B_{i}$ & & Parameter describing the pump in tube $i$. \\
\hline$C_{i}$ & & Parameter describing the pump in tube $i$. \\
\hline
\end{tabular}

\section{EQUATIONS}

The equations which describe solute and water movement in the model are $(1-4)$ for the loop of Henle and the collecting duct [1].

$$
\frac{d F_{i v}}{d x}=-S_{i} J_{i v}
$$




$$
\begin{gathered}
J_{i v}=l_{p i} \sum_{j} \sigma_{i j}\left(c_{4 j}-c_{i j}\right), \\
\frac{d F_{i j}}{d x}=-S_{i} J_{i j}, \\
J_{i j}=\left(l-\sigma_{i j}\right) \frac{\left(c_{4 j}+c_{i j}\right)}{2} J_{i v}-k_{i j}\left(c_{4 j}-c_{i j}\right)+T_{i j}, \\
T_{i j}=\frac{C_{i} c_{i j}}{A_{i}+c_{i j}+B_{i} c_{4 j}} .
\end{gathered}
$$

The equations for the central core are (5) and (6).

$$
\begin{gathered}
\frac{d F_{4}}{d x}=-\sum_{i=1}^{3} \frac{d F_{i v}}{d x}, \\
\frac{d\left(F_{4} c_{4 j}\right)}{d x}=-\sum_{i=1}^{3} \frac{d\left(F_{i v} c_{i j}\right)}{d x} .
\end{gathered}
$$

\section{BOUNDARY CONDITIONS}

The model is described by twelve ordinary first order differential equations, so we need twelve boundary conditions. These have been derived in [1] and are listed below in Table 2.

TABLE 2

Boundary Conditions for the Central Core Model

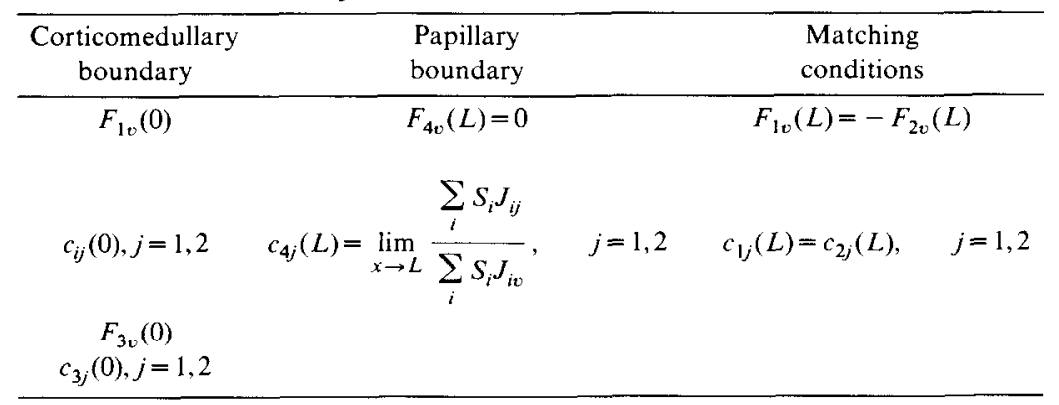

\section{PARAMETER AND INPUT VALUES}

In Table 3 we list the parameter values chosen for the initial runs. As we mentioned in the previous paper, there is a linear transition zone between the outer and inner medulla; we have chosen this to lie between 0.4 and 0.6 $\mathrm{cm}$. Thus any parameter value that changes between the outer and inner 
TABLE 3

Parameter and Input Values for Base Case

\begin{tabular}{|c|c|c|c|c|c|c|}
\hline \multirow[b]{3}{*}{ Tubule: $i=$} & \multicolumn{3}{|c|}{ Outer zone } & \multicolumn{3}{|c|}{ Inner zone } \\
\hline & DHL & $\begin{array}{l}\text { THICK } \\
\text { AHL }\end{array}$ & $\mathrm{CD}$ & DHL & $\begin{array}{l}\text { THIN } \\
\text { AHL }\end{array}$ & $\mathrm{CD}$ \\
\hline & 1 & 2 & 3 & 1 & 2 & 3 \\
\hline & \multicolumn{3}{|c|}{$\left(L_{1}=0.5 \mathrm{~cm}\right)$} & \multicolumn{3}{|c|}{$\left(L_{2}=1.0 \mathrm{~cm}\right)$} \\
\hline$S_{i}\left(\mathrm{~cm}^{2} / \mathrm{cm}\right)$ & 800 & 800 & 800 & 800 & 800 & 800 \\
\hline $\mathrm{RTL}_{\rho i}$ & 0.25 & 0 & $0.02(\mathrm{ADH})$ & 0.25 & .0015 & $0.02(\mathrm{ADH})$ \\
\hline$\left(\mathrm{cm}^{4} /\right.$ mmole-min $)$ & & & $0.003($ No $A D H)$ & & & $0.003\left(\mathrm{~N}_{0} \mathrm{ADH}\right)$ \\
\hline$\sigma_{i 1}(\mathrm{salt})$ & 0.96 & 1.0 & 1.0 & 0.96 & 0.9 & 1.0 \\
\hline $\begin{array}{l}k_{i 1} \text { (salt) } \\
(\mathrm{cm} / \mathrm{min})\end{array}$ & .001 & 0 & .00004 & .001 & .015 & .00004 \\
\hline$\sigma_{i 2}$ (urea) & 0.95 & 1.0 & 1.0 & 0.95 & 0.9 & 1.0 \\
\hline $\begin{array}{l}k_{i 2} \text { (urea) } \\
(\mathrm{cm} / \mathrm{min})\end{array}$ & .001 & 0 & .00015 & .001 & .004 & .00015 \\
\hline Pump parameters & & & & & & \\
\hline$A_{i}$ & - & 0.5 & - & - & 0.5 & - \\
\hline$R_{i}$ & - & 0.5 & - & 一 & 0.5 & $\ldots$ \\
\hline$C_{i}$ & - & .0075 & - & - & $0^{\mathrm{a}}$ & - \\
\hline Input values & & & & & & \\
\hline$F_{i v}\left(\mathrm{~cm}^{3} / \mathrm{min}\right)$ & 2.0 & - & 0.5 & - & - & - \\
\hline$c_{i 1}(\mathrm{mM})$ & 285 & - & 100 & - & - & - \\
\hline$c_{i 2}(\mathrm{mM})$ & 15 & 一 & 200 & - & - & - \\
\hline
\end{tabular}

${ }^{\text {a }}$ For $C_{2}=0$ in thin AHL the pump activity is zero. Setting this $C_{2}$ to a positive number introduces pump activity into the thin AHL.

zones is changed linearly from the value given for the outer zone to that for the inner zone over this region. The estimation of the values for $S_{i}$ have also been discussed in [1].

The thermodynamic parameters come from the literature. In almost all instances, they were obtained from studies on isolated perfused tubules. Notice that with the exception of the AHL, these parameters remain constant over the length of the tubule. This fact is probably most important when considering the collecting duct, since it is generally felt that the outer portion of the CD is impermeable to salt and urea whereas the inner portion is permeable, especially to urea. Unfortunately, the data available are for the cortical and outer medullary portions; we have used these. One should also notice that the difference between the presence and absence of ADH appears only in the permeability properties of the CD.

The pump parameters were chosen arbitrarily, but we required that the salt concentration leaving the top of the AHL lie close to zero and that the pump acted in a uniform fashion over the length of the thick AHL. We have set the salt permeability equal to zero in this portion of the AHL 
because we believe that the pump will overpower any passive salt movement. We have also set the urea permeability equal to zero reflecting our belief that there is little passive movement of urea across the walls of the thick AHL.

In Table 3 we also list the initial values for flow rates and concentrations into the top of the DHL and $\mathrm{CD}$ for the initial runs. The problems in estimating these values have been discussed in [1].

\section{COMPUIATIONAL METHODS}

The method of quasilinearization [2] was used to integrate the set of simultaneous, first order differential equations of the central core model. Quasilinearization is an iterative linearization method of the Newton-Raphson type for two point boundary value problems in systems of nonlinear differential equations. Basically, the method involves guessing initial solutions that satisfy the boundary conditions, linearizing the differential equations by expanding in Taylor's series around the guessed solutions, and retaining only first order terms. The domain of the problem is then divided into $n$ intervals, and the derivatives and function values are approximated by finite differences. From this, one obtains a set of simultaneous linear algebraic equations which can be solved by standard computational techniques to give a corrected set of solutions. This process is then repeated until convergence meets preset criteria or it is clear that the solution process is not converging. We have generally required that successive iterates differ by less than $10^{-3}$ of their values at all points.

Of the several possible ways to formulate our equations for quasilinearization, we have chosen the following. We first rewrite Eqs. (1) and (3) for tube $i$ as (7-9) in which $f_{i v}, f_{i 1}$ and $f_{i 2}$ may be considered to be functions of the concentrations along. Let $F_{i v}^{0}, c_{i 1}^{0}, c_{i 2}^{0}$ be the

$$
\begin{array}{r}
\frac{d F_{i v}}{d x}=f_{i t}, \\
\frac{d\left(F_{i t} c_{i 1}\right)}{d x}=f_{i 1}, \\
\frac{d\left(F_{i v} c_{i 2}\right)}{d x}=f_{i 2},
\end{array}
$$

current guesses for the functions $F_{i t}, c_{i 1}$ and $c_{i 2}$ which satisfy the boundary conditions. For our initial guesses we have generally taken linear functions of $x$ which match the boundary conditions. We can then write $F_{i v}, c_{i 1}$ and $c_{i 2}$ in terms of corrections to the guessed solutions, $\varepsilon_{i v}, \varepsilon_{i 1}, \varepsilon_{i 2}$, as in (10-12). 
Expanding the right hand sides of (7) and (8) around the current

$$
\begin{aligned}
& F_{i v}=F_{i v}^{u}+\varepsilon_{i v}, \\
& c_{i 1}=c_{i 1}^{0}+\varepsilon_{i 1}, \\
& c_{i 2}=c_{i 2}^{0}+\varepsilon_{i 2},
\end{aligned}
$$

approximations to the solutions we obtain (13) and (14). Equation (9)

$$
\begin{array}{r}
\frac{d\left(F_{i v}^{0}+\varepsilon_{i v}\right)}{d x}=f_{i v}^{0}+\sum_{i=1}^{4}\left[\frac{\partial f_{i v}^{0}}{\partial c_{i 1}} \varepsilon_{i 1}+\frac{\partial f_{i v}^{0}}{\partial c_{i 2}} \varepsilon_{i 2}\right] \\
\frac{d\left[\left(F_{i v}^{0}+\varepsilon_{i v}\right)\left(c_{i 1}^{0}+\varepsilon_{i 1}\right)\right]}{d x}=f_{i 1}^{0}+\sum_{i=1}^{4}\left[\frac{\partial f_{i 1}^{0}}{\partial c_{i 1}} \varepsilon_{i 1}+\frac{\partial f_{i 1}^{0}}{\partial c_{i 2}} \varepsilon_{i 2}\right]
\end{array}
$$

is handled in the same manner as (8) and will not be exhibited. In (13) and (14) $f_{i v}^{0}$ and $f_{i 1}^{0}$ and their derivatives are evaluated for the current values of the approximations to the solutions.

The length $L$ is divided into $n$ intervals, the $k$ th interval extending from $x_{k}$ to $x_{k+1}$. In place of using finite differences to approximate (13) and (14) directly, we integrate (13) and (14) over the $k$ th interval, using the trapezoid approximation to evaluate the right hand sides and then drop the quadratic term in the corrections on the left side of (14). This gives us Eqs. (15) and (16).

$$
\begin{aligned}
& \varepsilon_{i v, k+1}-\varepsilon_{i v k}+F_{i \mathrm{c}, k+1}^{0}-F_{i v k}^{0}=\frac{\Delta x}{2}\left\{f_{i \mathrm{c}, k+1}^{0}-f_{i v k}^{0}\right. \\
& \left.+\sum_{i=1}^{4}\left[\left.\frac{\partial f_{i v}^{0}}{\partial c_{i 1}}\right|_{k+1} \varepsilon_{i 1, k+1}+\left.\frac{\partial f_{i c}^{0}}{\partial c_{i 1}}\right|_{k} \varepsilon_{i 1 k}+\left.\frac{\partial f_{i v}^{0}}{\partial c_{i 2}}\right|_{k+1} \varepsilon_{i 2, k+1}+\left.\frac{\partial f_{i v}^{0}}{\partial c_{i 2}}\right|_{k} \varepsilon_{i 2 k}\right]\right\} \\
& \left(F_{i v}^{0} c_{i 1}^{0}+F_{i c}^{0} \varepsilon_{i 1}+c_{i 1}^{0} \varepsilon_{i 2}\right)_{k+1}-\left(F_{i v}^{0} c_{i 1}^{0}+F_{i c}^{0} \varepsilon_{i 1}+c_{i 1}^{0} \varepsilon_{i 2}\right)_{k} \\
& =\frac{\Delta x}{2}\left\{f_{i 1, k+1}^{0}+f_{i 1 k}^{0}+\sum_{i=1}^{4}\left[\left.\frac{\partial f_{i 1}^{0}}{\partial c_{i 1}}\right|_{k+1} c_{i 1, k+1}+\left.\begin{array}{c}
\partial f_{i 1}^{0} \\
\partial c_{i 1}
\end{array}\right|_{k} \varepsilon_{i 1 k}\right.\right. \\
& \left.\left.+\left.\frac{\partial f_{i 1}^{0}}{\partial c_{i 2}}\right|_{k+1} \varepsilon_{i 2, k+1}+\left.\frac{\partial f_{i 1}^{0}}{\partial c_{i 2}}\right|_{k} \varepsilon_{i 2 k}\right]\right\} \text {. }
\end{aligned}
$$


Note that Eqs. (15) and (16) are linear functions of the corrections $\varepsilon_{i j k}, \varepsilon_{i v k}$. Equations (5) and (6) for the central core were handled in a similar manner.

The boundary conditions that are known values or matching conditions are simply handled. Written in terms of the corrections at the boundaries, they give additional linear equations. The only problem is the limit boundary conditions for $c_{41}(L)$ and $c_{42}(L)$. We describe how $c_{41}(L)$ was handled; $c_{42}(L)$ was handled in the same way. This boundary condition can be written as in Eq. (17). Since $F_{4 v}=0$ at $x=L$, we use l'Hopital's

$$
c_{41}(L)=\operatorname{Lim}_{x \rightarrow L} \frac{F_{41}}{F_{4 v}},
$$

rule as in (18), assuming the limit of the ratio of derivatives exists.

$$
c_{41}(L)=\left.\frac{d F_{41} / d x}{d F_{4 v} / d x}\right|_{L}
$$

This is obtained directly if we write equations for the central core equivalent to (7) and (8), Eqs. (19) and (20). At $x=L, F_{4 v}=0$ and

$$
\begin{aligned}
\frac{d F_{4 v}}{d x} & =f_{4 v}, \\
\frac{d\left(F_{4 v} c_{41}\right)}{d x} & =F_{4 v} \frac{d c_{41}}{d x}+c_{41} \frac{d F_{4 v}}{d x}=f_{41},
\end{aligned}
$$

assuming $d c_{41} / d x$ is bounded, note that (20) evaluated at $x=L$ is the same as (18). Substituting from (19) gives Eq. (21). If Eq. (21) is

$$
\left(c_{41} f_{4 v}-f_{41}\right)_{x=L}=0,
$$

now expanded in terms of corrections, $\varepsilon_{i j}(L)=\varepsilon_{i j, n+1}$, and linear terms in corrections retained we have the boundary conditions corresponding to the limit boundary conditions at $x=L$.

Using the above approximations we obtain a large system of linear algebraic equations which is solved by standard methods to obtain the corrections. The guesses for the solutions at the mesh points of interval $L$ are corrected and the process is repeated. We have used sixteen intervals over $L$ for production runs and eight intervals for exploratory work. As a check that the grid size was not too coarse, a few of the simulations were repeated with twenty-four intervals; the results obtained did not differ by more than a few percent from those obtained with sixteen intervals.

A problem which caused us considerable trouble initially was the occurrence of damped oscillations that were centered around the boundary between the inner and outer zones of the medulla, i.e., between $L_{1}$ and $L_{2}$. 
This difficulty was traced to discontinuities or large changes over small distances in some of the parameter values giving rise to either discontinuities or very large values in some of the derivative evaluations on crossing the boundary between $L_{1}$ and $L_{2}$. These oscillations disappeared or became much smaller when we replaced all discontinuities in parameter values by linear transitions between the two values of each parameter that extended from $L_{11}$ to $L_{22}$. The program was written so we could change the length of the transition region; this facility was later used to introduce a linear gradient in properties of the tubules that extended over almost the entire medulla.

The program was written in FORTRAN IV and run on the MTS system on the IBM 360/67 at the University of Michigan Computing Center.

\section{RESULTS OF THE SIMULATIONS}

\section{THE BASIC RABBIT}

In Figs. 2-5 we show the profiles for the volume flow rate, total concentration in the central core, and solute concentrations generated when the equations describing the model are solved using the parameter and input values listed in Table 3. The two cases when ADH is present and absent respectively are shown in Figs. 2 and 3 whereas the profiles given in Figs. 4 and 5 are for the case when $\mathrm{ADH}$ is present. We have arbitrarily called the latter case, the one where ADH is present, the basic rabbit. In Figs. 4 and 5 the concentration profile for the central core is not shown. In both cases, the concentration in the central core is almost equal to that in the DHL from the corticomedullary border to a depth of $0.4 \mathrm{~cm}$. Between 0.4 and $0.6 \mathrm{~cm}$ the profile crosses over to that for the AHL, and then the concentration in the central core is essentially equal to that in the AHL from $0.6 \mathrm{~cm}$ to the tips of the papillae.

Several observations are immediately obvious. First and foremost is the fact that a concentration gradient is established in the central core. Figures 3-5 show this for total concentration, salt and urea respectively. Second and equally important is the fact that the fluid leaving the $\mathrm{CD}$ is more concentrated than that entering. Moreover, the concentration and relative composition of this fluid is well within the limits for those values determined experimentally. Third is the fact that water is abstracted from the DHL. It is important to notice from Fig. 2 that most of the abstraction occurs in the first part of the DHL, a prediction of the model which is in agreement with Pennell [3]. Also, water is abstracted from the $\mathrm{CD}$, and the urine flow rate, $F_{3 v}(L)$, agrees with those values which have been measured on the rabbit. Observe, too, that the volume flow rate of fluid leaving the top of the AHL is just slightly larger than the inflow rate into the CD, $F_{30}(0)$. 


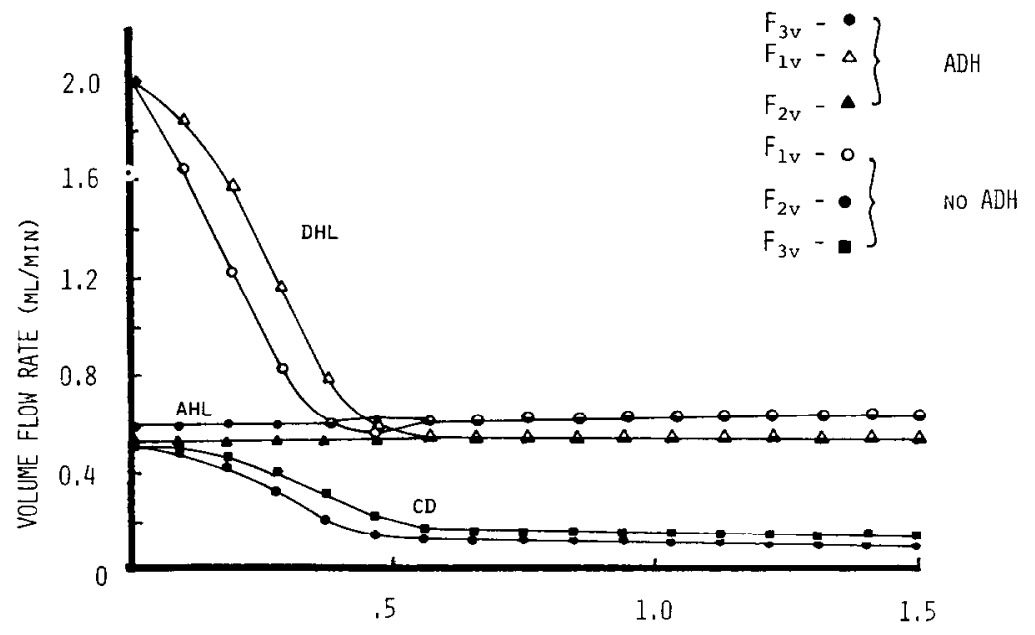

DISTANCE INTO MEDULLA (CM)

FIG. 2. Volume flow rates obtained in presence and absence of ADH in model of basic rabbit. Transition zone extends from $0.4 \mathrm{cml} 100.6 \mathrm{~cm}$.

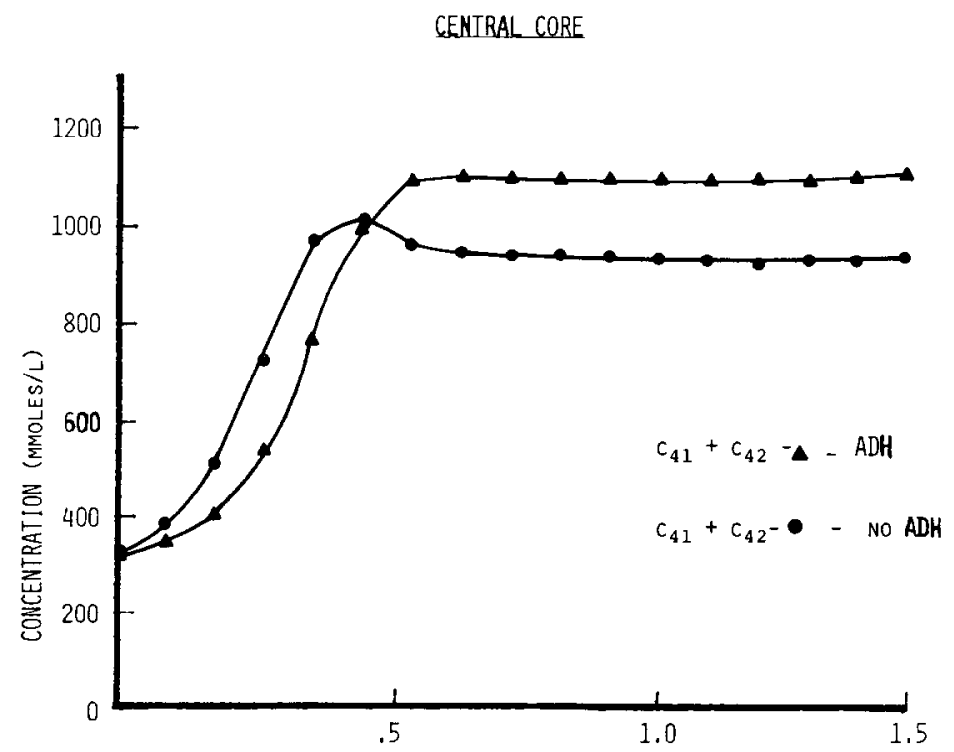

DISTANCE INTO MEDULLA ( $\mathrm{CM}$ )

FIG. 3. Concentration gradient developed in central core in basic rabbit model. 


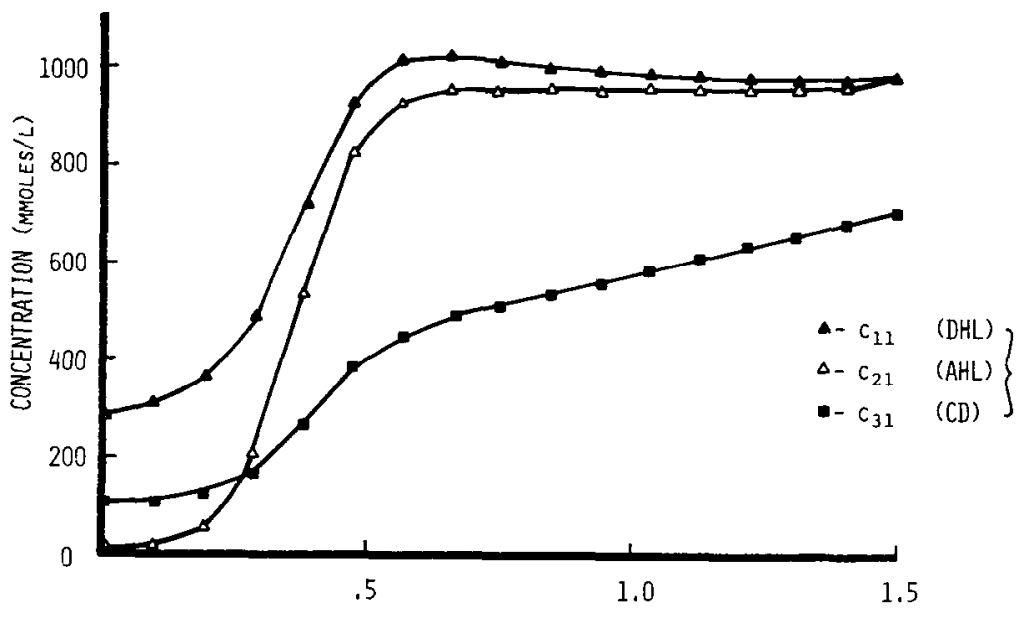

DISTANCE INTO MEDULLA (cM)

FIG. 4. Salt concentration profiles in basic rabbit model in presence of ADH.

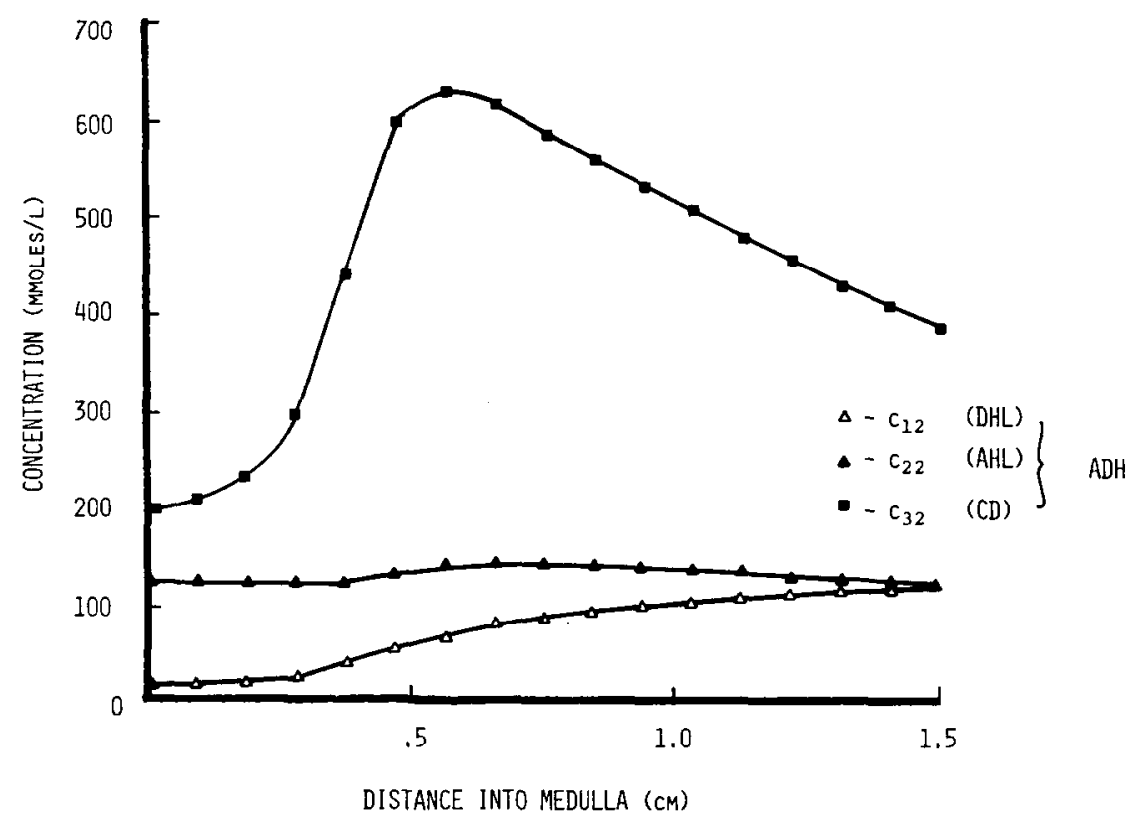

FIG. 5. Urea concentration profiles in basic rabbit model in presence of ADH. 
It is important to examine Figs. 2-5 in detail for it is from them that one can see how the concentration gradients are established. In Figs. 3-5 we see that the sharp rise in total concentration in the DHL, the CD, and the central core occurs primarily in the outer medulla. From Fig. 2 we see that this rise occurs concomitantly with a decrease in the volume flow rate in the DHL and CD suggesting that water abstraction is playing the major role in these two tubules over this region. The water abstraction is due to the osmotic activity of the salt being pumped out of the thick AHL, and the concomitant rise in the salt concentration in the DHL as seen in Fig. 4 is due to the fact that the reflection coefficient for salt is close to one together with the fact that the salt permeability is low.

Throughout the inner medulla the total concentration in the central core and the volume flow rates remain essentially constant. Since the volume flow rate changes very little in this zone, the changes seen in the salt and urea concentration profiles must reflect shifts in the solutes between the tubules and the central core. Thus the gradual decrease in urea in the CD in the inner zone is a movement of urea into the central core and then into the DHL and, to a lesser extent, the thin AHL. The increase in urea in the AHL is eventually delivered to the $\mathrm{CD}$ again, so the model does predict urea recycling.

There are two important observations which must be made at this point. One is that even though the urea recycles, it does not contribute to a significant buildup of the concentration gradient in the inner zone. Specifically, it has been predicted by Kokko and Rector [4] and Stephenson [5] and Stephenson et al. [6] that a significant increase in the urea concentration in the medullary interstitium in the inner zonc would result in the abstraction of water from the DHL. The result would be a salt concentration in the lower portion of the thin AHL which would exceed that in the interstitium. With the thin AHL highly permeable to salt, the salt would then diffuse out, thereby generating the concentration gradient in the inner zone. Thus the recycling of urea is the driving force for this predicted generation of a concentration gradient in the absence of active transport. What we see in Fig. 3 is that there is a small buildup of the concentration gradient in the inner zone, but with the parameter and input values used for the basic rabbit, the osmotic activity of the urea is not seen, i.e., in Fig. 4 we see that the concentration of salt in the thin AHL is essentially the same as that in the central core and is less than that in the DHL.

The second observation is that the loss of urea from the CD is almost made up by an entry of salt into the CD. The result is that virtually no change in the total concentration of the fluid in the $C D$ is seen as it passes through the inner zone.

Finally two points are worth mentioning about the ADH effects. First is that the changes in the various profiles seen as a result of the presence or 
absence of ADH are quantitative and not qualitative, i.e., the net effect is simply to shift the curves. Second is that in the presence of ADH we expect a urine concentration that is somewhat higher than that predicted by the model and in the absence of ADH we expect a higher flow rate and lower concentration than is given by these simulations. We remind the reader that the only difference between the cases when ADH is present or absent is in the water filtration in the $\mathrm{CD}$, i.e., we have not adjusted the volume inflow rate into the $\mathrm{CD}$ or adjusted the entering concentrations of solute, both of which we would expect to change as a result of the action of ADH on the distal tubule.

\section{GEOMETRIC STUDIES AND PUMP STUDIES}

If we review what parameter and input values are needed for the model, we see that the areas of major concern are the geometric parameters and the pump parameters.

The form for the pump term, $T_{i 1}$, has been discussed in [1]. It remained for us to select values for $A_{i}, B_{i}$, and $C_{i}$. To do this, we set all the other parameter and input values equal to those listed in Table 3 , and then executed a series of simulations in which $A_{i}, B_{i}$, and $C_{i}$ were varied. The criteria which we set to select the values for these parameters given in Table 3 were that the salt concentration leaving the top of the thick AHL must be close to zero, and that the salt concentration in the thick AHL must decrease gradually over its length.

As a result of these simulations we can make two observations. One is that if we look only at the urine volume flow rate, $F_{3 v}(L)$, and concentrations $c_{31}(L)$ and $c_{32}(L)$, what is important is that salt be removed from the thick AHL. We found that if the pump was so strong as to effectively remove all of the salt over the first portion of the thick AHL, the values for the urine volume flow rate and concentration were practically the same as those for the basic rabbit. The other is that the generation of the concentration gradient in the central core is very much dependent upon the strength of the pump. Thus if the pump is weak and does not remove all of the salt from the thick AHL, the concentration gradient in the central core is less than that of the basic rabbit.

Another area of concern is the surface area per unit length of the various tubular segments. The estimates for these values for the DHL and thin AHL are probably fairly good as is the value for the thick AHL. The estimate for the $C D$ is probably not reliable. To investigate the effect the latter two have on the urine volume flow rate and concentration, we varied these surfaces in turn keeping all other parameter and input values equal to those given in Table 3.

Changing the surface area per unit length of the thick AHL to 1000 $\mathrm{cm}^{2} / \mathrm{cm}$ had no effect on urine concentration or flow rate. Increasing $S_{3}$ to 
$1000 \mathrm{~cm}^{2} / \mathrm{cm}$ decreased urine flow rate and urea concentration slightly and increased salt concentration slightly. Decreasing $S_{3}$ to $600 \mathrm{~cm}^{2} / \mathrm{cm}$ had similar small effects in the opposite direction. In all cases, the values obtained were within the bounds for those values determined experimentally. Thus the model is not highly sensitive to changes in $S_{2}$ and $S_{3}$ of $20-25 \%$.

A word of caution is in order at this point. The geometry of the kidney is such that the medulla is pyramidal in shape. For this reason, one might argue that the surface area per unit length should decrease as one goes from the corticomedullary border to the tips of the papillae. This would reflect, among other things, the fact that not all long Henle loops are of the same length. Since the model does not include loops of Henle of different lengths, we have not carried out any detailed studies involving a decrease in surface area as a function of the depth into the medulla.

The final study in the series to determine the geometric parameters for the basic rabbit involves the selection of $L_{11}$ and $L_{22}$, i.e., the beginning and the end of the transition 7one between the outer and inner medulla. We discussed in the previous section the problems with the occurrence of damped oscillations in the numerical solutions centered in this region. The values of $0.4 \mathrm{~cm}$ and $0.6 \mathrm{~cm}$ for $L_{11}$ and $L_{22}$, respectively, came about as a compromise. On the one hand, as we let these values approach $0.5 \mathrm{~cm}$, the oscillations increased. On the other hand, if we let these values go far from $0.5 \mathrm{~cm}$, we fecl the model is departing from what is reasonablc physiologically since the evidence suggests that the division between the outer and inner zone is fairly sharp.

To show how the profiles change, Figs. 6-8 correspond to Figs. 3-5 but with the transition zone extending from $0.4 \mathrm{~cm}$ to $1.4 \mathrm{~cm}$. As a result, the salt pump extends far down the thin AHL with the capacity decreasing as the papillae are approached. The total pump capacity is somewhat larger in this simulation than for the basic rabbit so the volume outflow from the AHL is now less than the inflow into the CD. Although the shapes of the profiles are changed considerably, the overall effect in producing a concentrated urine is much the same.

\section{INPUT-OUTPUT STUDIES}

The results which we have just presented, together with those of Stephenson $[5,7,8]$ and Stephanson et al. [6] confirm that the central core model is a viable model of the concentrating mechanism of the rabbit kidney. Because of this, we felt that it was important to carry out input-output studies to see how the model reacted to changes to inflow rates and concentrations into the DHL and CD.

In the first series of studies we investigated the effect that the composition of the fluid entering the DHL and CD had on the final composition 


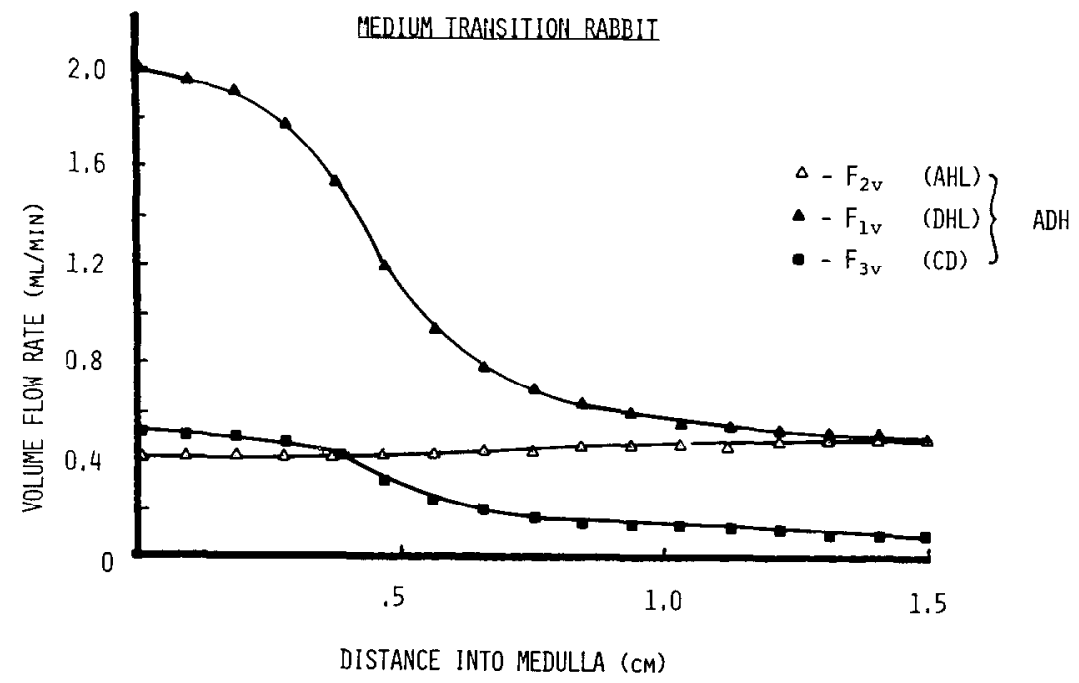

Fig. 6. Volume flow rates in model of basic rabbit with transition zone from $0.4 \mathrm{~cm}$ to $1.4 \mathrm{~cm}$.

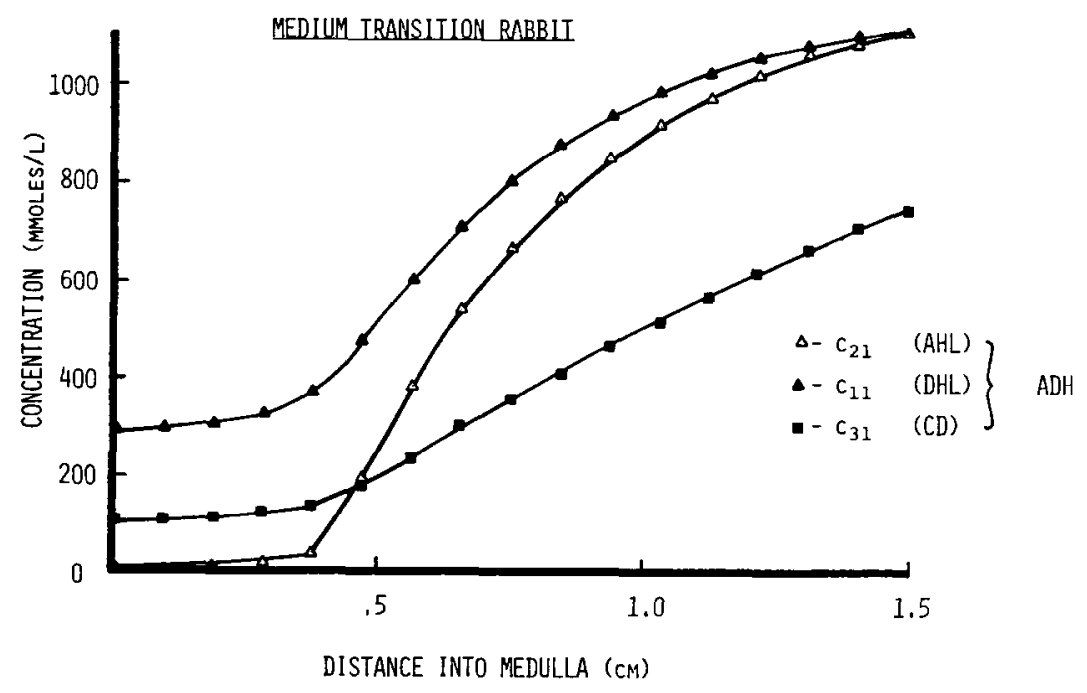

FIG. 7. Salt concentration profiles in model of basic rabbit with transition zone from $0.4 \mathrm{~cm}$ to $1.4 \mathrm{~cm}$. 


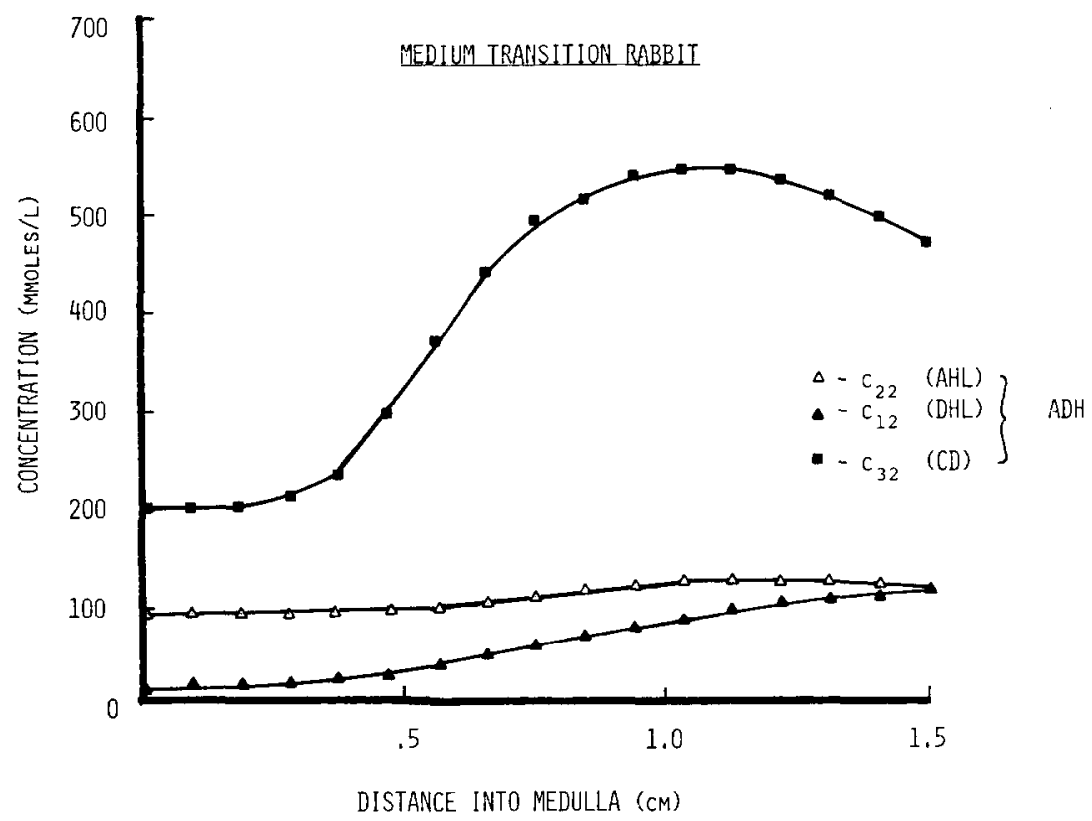

FiG. 8. Urea concentration profiles in model of basic rabbit with transition zone from $0.4 \mathrm{~cm}$ to $1.4 \mathrm{~cm}$.

and volume flow rate of the fluid leaving the CD. In the first study, we kept all parameter and input values equal to those given in Table 3 except for the concentration of salt and urea entering the DHL. Here we kept the total concentration equal to $300 \mathrm{mmole} / L$ but varied the distribution between the two solutes. The results are shown in Fig. 9. Note there is practically no effect on the urine volume flow rate and urea concentration but that the urine salt concentration decreases slightly as the salt concentration entering the DHL decreases. The point is that the concentrating engine does not depend upon the urea concentration entering the DHI. If no urea is present at all, however, the concentrating engine cannot produce a concentrated urine. The results of a simulation which was run when there was no urea present in the inflow into the $D H L$ and $C D$ are indicated on the ordinate in Fig. 9 and show that this is the case. Thus urea plays an essential role in establishing the concentration gradient but its role is passive and is initiated from the CD.

Figure 10 shows the results of a similar study in which the composition of the fluid entering the CD was changed. There is practically no effect on 


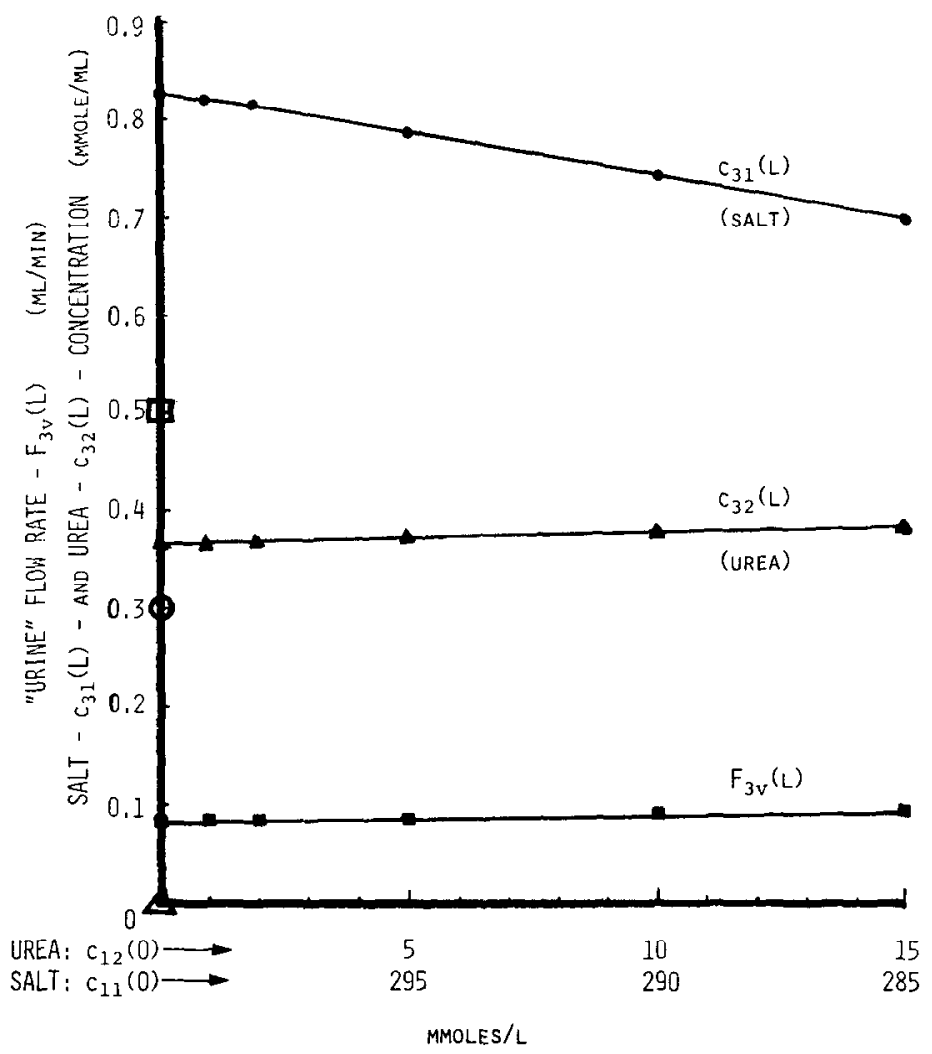

Fig. 9. Effect of changing composition of inflow into DHL on volume flow and concentrations of urine. The open symbols $\square, O$, and $\triangle$ on the ordinate are $F_{3 v}(L)$, $c_{31}(L)$ and $c_{32}(L)$, respectively, when no urea is included in inflows to DHL and CD.

the urine volume flow rate and total concentration. The changes in the relative composition of the urine simply reflect the changes in the input into the CD.

This series of studies underlines the importance of the concentrating mechanism being at least a two solute system in which one solute is actively extruded from the thick AHL and the other is allowed to function passively. Given these facts, it is possible to examine the role that active transport in other tubular segments would play in the system. We have already seen what effect is generated when there is pumping activity in the thin AHL. 


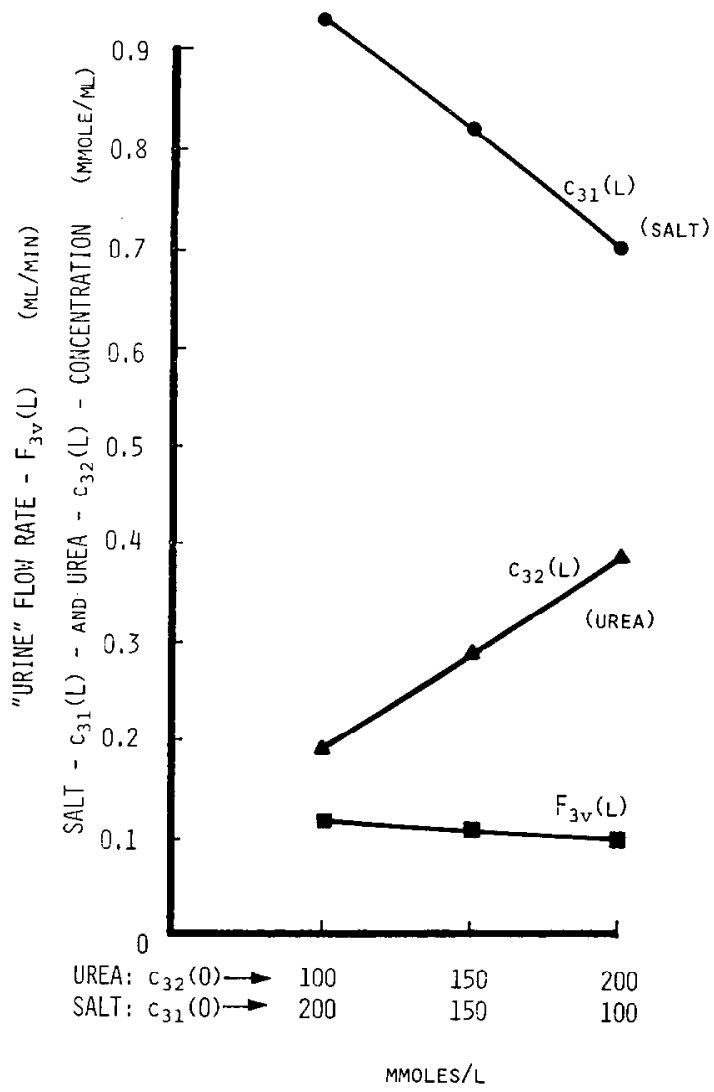

FIG. 10. Effect of changing composition of inflow to $\mathrm{CD}$ on volume flow and concentrations of urine.

Some preliminary studies have been done on the effect of salt pumping in the upper and lower portions of the CD. The net result so far is that it is possible to generate just about any gradient and profile desired with the appropriate placement of pumps. We feel, however, that this does little towards answcring the qucstions about the possible existence of pumps in the thin AHL or CD.

The second series of input-output studies involved changing the inflows to the DIHL and CD, and seeing what effect this had on the urine flow rate and concentration. Figure 11 shows the effect of changing the inflow into the DHL, $F_{1 v}(0)$. When $F_{1 v}(0)=0$, no salt is delivered to the pumps in the 
thick AHL so there cannot be effective concentration; the fluid entering the $\mathrm{CD}$ leaves it unchanged. As $F_{1 v}(0)$ increases, we see that the total urine concentration rises and the urine volume flow rate decreases reflecting the increased delivery of salt to the pumps. The rise would probably differ in real kidneys with the vasa rectae present, but the same phenomenon would have to be present if one could change only $F_{1 i}(0)$. As can be seen from the

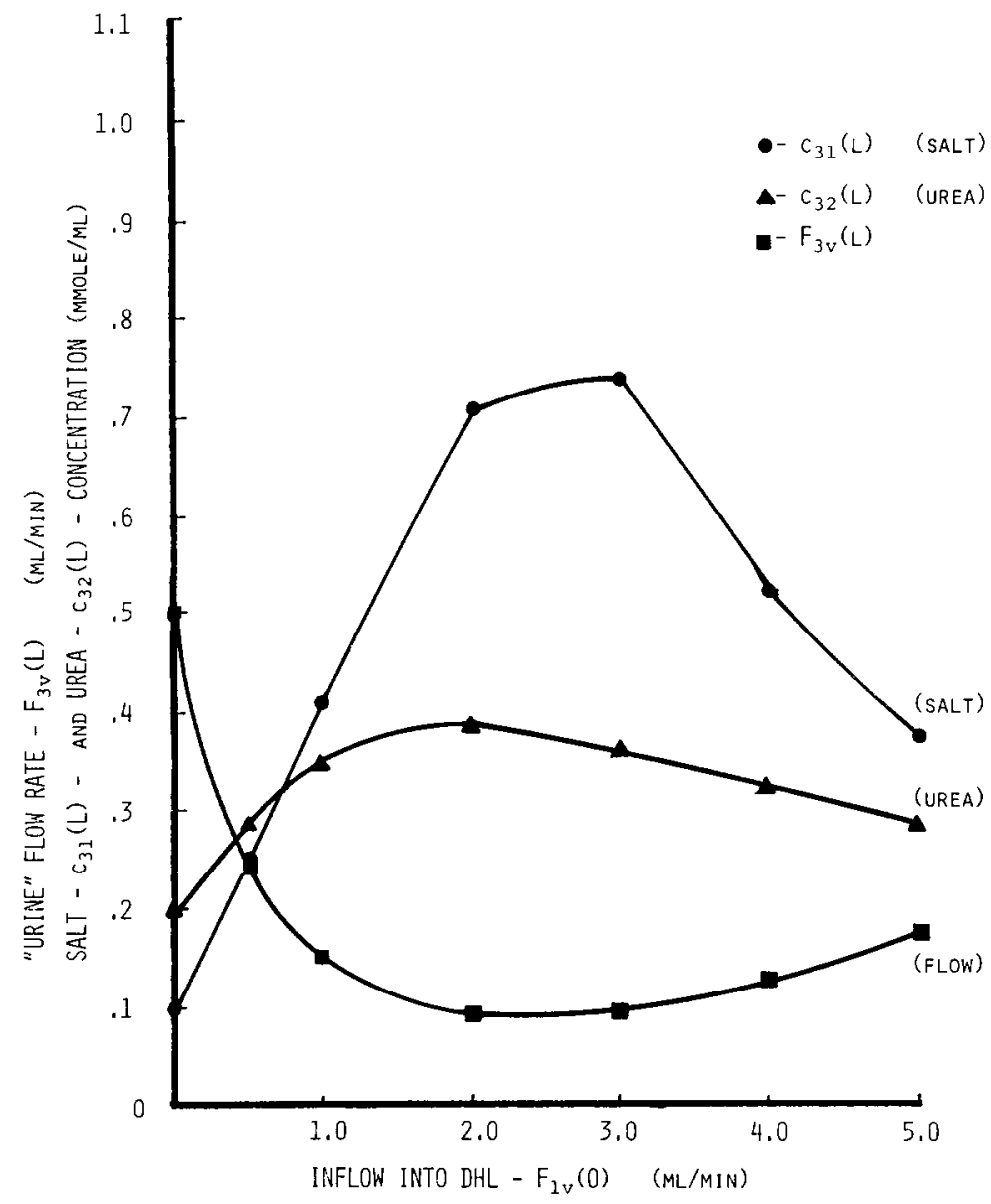

FIG. 11. Effect of changing volume inflow to the DHL on urine volume flow and concentrations for basic rabbit model. The composition of the inflow to DHL is constant, $c_{11}=285, c_{12}=15 \mathrm{mM}$. 
figure, as $F_{1 v}(0)$ increases still further the urine concentration plateaus and eventually falls. As we point out in the discussion of the limit cases in the previous paper [1] this is due to two effects. One is the saturation of the pumping capacity for a given salt concentration entering the thick AHL, the other is due to a decrease in salt concentration entering AHL when inflow to DHL becomes large enough.

In interpreting the results from this study, it is important to remember that in this version of the central core model, the volume flow rate leaving the top of the AHL is not coupled to that entering the CD. We have done this deliberately since we wanted to be able to examine responses of the model to changes in the input values into the DHL separately from those for the CD.

The physiological significance of the results found in the present study depends on the range of inflow rates into the DHL that can be obtained in a real kidney for given constant inflow into the $\mathrm{CD}$. The inflow rate into the $\mathrm{CD}, \mathrm{F}_{3 \mathrm{v}}(0)$, is determined by the action of both the cortical nephrons and the deep nephrons. For those species like the rabbit whose nephrons are not all long-looped the coupling between outflow from the AHL of the long medullary Henle loops and inflow into the CD may be quite loose because a significant portion of the inflow into the CD comes from cortical nephrons which have small Henle loops that barely reach the medulla. For those species whose nephrons are all long-looped we would expect a tighter coupling between the flow rate leaving the top of the AHL and that entering the $\mathrm{CD}$, but this coupling would be modulated by what happens along the distal tubule, and even this depends upon the particular state of the kidncy.

The final study in this series involved changing the inflow rate to the CD. The changes, shown in Fig. 12, are in the expected direction. Again, the physiological interpretation of this study must be tempered by the preceding remarks.

\section{DISCUSSION AND CONCLUSIONS}

In this paper we have presented the results of simulations using the central core model. We have seen that if we use those parameter and input values which have either been determined experimentally or estimated based upon experimental evidence from the rabbit kidney, then the central core model predicts that a concentration gradient is generated in the central core and a concentrated urine is produced. The concentration gradient, and urine volume flow rate and concentration which are predicted lie well within the bounds for those values which have been measured experimentally. Moreover, the shift in the profiles in the presence or absence of ADH is in the direction which one would expect. 


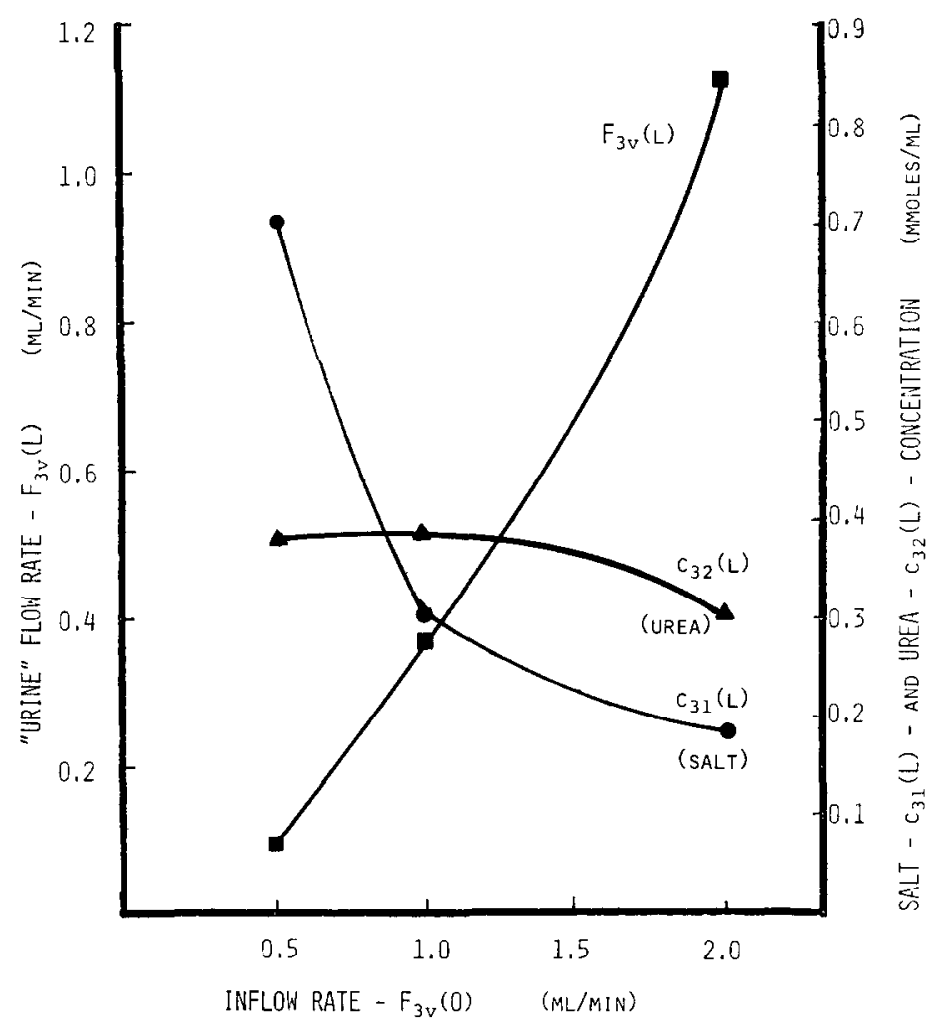

FIG. 12. Effect of changing volume inflow to $C D$ on urine volume flow and concentrations for basic rabbit model.

An important observation to be made based upon the basic rabbit concerns the phenomenon of urea recycling. With the parameter and input values listed in Table 3, urea recycling was predicted, but the expected osmotic effect in the inner medulla was not seen. It is interesting to note, however, that this osmotic effect which would be necessary to generate a concentration gradient in the inner medulla is not needed for the model to predict concentration gradients which lie within physiological bounds.

The input-output studies which we have done show how the model behaves when subjected to changes in these values. They produced no surprises, but did underline the importance of urea in the concentration mechanism in a model for this type. Moreover, the studies on the volume 
inflow rate into the DHL and CD bore out our conclusions in [1] in the discussion of the limit cases.

From the point of view of how simulations using the central core model can contribute to our understanding of the mechanisms involved in the control of urine flow rate and concentration, the most interesting study is the one where the volume inflow rate into the DHL is varied. The response as seen in Fig. 11 is biphasic, and is so striking that one is compelled to ask whether and how it might be involved in the control of urine concentration. The reasonable range of values for inflow into the $\mathrm{DHL}$ is probably that for the rising limb of the curve of concentration, for normal operation.

What are the factors involved in the control of urine concentration? Aside from the action of ADH on water permeability, it is generally thought that medullary blood flow plays a major role in determining the urine concentration. As early as 1958 Berliner et al. [9] pointed out that medullary blood flow had to be low for urine to be concentrated, whereas in the diuretic state, medullary blood flow increased [10]. Thurau and Deetjen [11] and Thurau, Deetjen, and Kramer [12] have shown that with an increase in pressure the blood flow in the medulla is increased, and they suggested that the increased blood flow washes out the concentration gradient in the medulla. This conclusion coincides with our argument in [1] concerning increased flow in the vasa rectae.

It is clear that the volume flow rate in the vasa rectae and loops of Henle are intimately involved in the operation of the countercurrent multiplier. The question is how are they interrelated? For a given flow rate in the afferent arterioles, increasing the resistance of the efferent arterioles should decrease the flow in the vasa rectae and increase the filtration rate and as a result, increase the inflow into the DHL. Thus the action of efferent arteriolar resistance is to couple these two determinants of the countercurrent concentration gradient so they act in concert. On the other hand, for a given resistance in the efferent arterioles, increasing the resistance of the afferent arterioles should decrease both the flow in the vasa rectae and the filtration rate. The effect of afferent arteriolar resistance is to couple the two so they act in opposition. If the afferent and efferent arteriolar resistances could be controlled independently this would provide a mechanism for independently controlling the flow in the vasa rectae and inflow into the DHL.

Is there any evidence for control of the components of this mechanism? Davis and Schnermann [13] have proposed that ADH acts indirectly to control resistance of the vasa rectae, the argument being that the effective changes in postglomerular resistance are due to changes in blood viscosity which depend on the concentration gradient attained in the medulla. Indeed, ADH is known to affect blood flow to the medulla. Fourman and Kennedy [14] and Thurau et al. [12] have both found a decrease in blood 
flow in the vasa rectae during antidiuresis. Fourman and Kennedy [14] go on to suggest that ADH constricts the efferent arterioles of the juxtamedullary glomeruli. Recently, Johnson et al. [15] have found an increase in the fraction of blood flow to the cortical region next to the medulla compared to that of the outer cortex in dogs injected with ADH. These findings suggest that ADH may play a direct role in control of efferent arteriolar resistance. Whether independent mechanisms exist for control of afferent and efferent arteriolar resistance and how $\mathrm{ADH}$ fits into that, is yet to be seen.

We wish to thank Dr. James A. Schafer and Dr. John Stephenson for their help in critically reviewing earlier versions of this and the preceding paper.

After these studies were completed Dr. Schafer pointed out that some better and different estimates of some of the thermodynamic parameters are row available. The major changes are that $R T L_{p}$ should be larger in the $C D$ and that urea permeability is larger for the $C D$ in the inner zone. In preliminary studies we have found that the results are not changed qualitatively by these changes but that the effect of the first change is to increase both salt and urea concentrations in urine, but that the effect of the second is to increase urine salt and decrease urine urea concentrations. Details of the results with these updated parameter estimates will be published in another paper.

\section{REFERENCES}

I J. A. Jacquez, D. Foster and E. Daniels, Solute concentration in the kidney-I. A model of the renal medulla and its limit cases, Math. Boisci. 32 (3/4), 307-336 (1976); this issue, preceding paper.

2 R. Bellman and R. Kalaba, Quasilinearization and Nonlinear Boundary-Value Problems, American Elsevier Publ. Co., Inc., New York, 1965.

3 J. P. Pennell, V. Sanjana, N. K. Frey and K. L. Jamison, The effect of urea infusion on the urinary concentrating mechanism in protein-depleted rats, J. Clin. Invest. 55, 399-409 (1975).

4 J. P. Kokko and F. C. Rector, Jr., Countercurrent multiplication system without active transport in inner medulla, Kidney Internat. 2, 264-270 (1972).

5 J. L. Stephenson, Concentration of urine in a central core model of the renal counterflow system, Kidney Internat. 2, 85-94 (1972).

6 J. L. Stephenson, R. P. Tewarson and R. Majia, Quantitative analysis of mass and energy balance in non-ideal models of the renal counterflow system, Proc. Nat. Acad. Sci., USA 71, 1918-1622 (1974).

7 J. L. Stephenson, Concentrating engines and the kidney-I. Central core model of the renal medulla, Biophys. J. 13, 512-545 (1973).

8 J. L. Stephanson, Concentrating engines and the kidney-II. Multisolute central core systems, Biophys. J. 13, 546-567 (1973).

9 R. W. Berliner, N. G. Levinsky, D. G. Davidson and M. Eden, Dilution and concentration of the urine and the action of antidiuretic hormone, Am. J. Med. 24, $730-743$ (1958). 
$10 \mathrm{H}$. Wirz and R. Dirix, Urinary concentration and dilution, Ch. 13, Handbook of Physiology, Sect. 8, Renal physiology, J. Orloff and R. W. Berliner, eds., Am. Physiol. Soc., Washington, DC, 1973.

11 K. Thurau and P. Deetjen, Die Diurese bei arteriellen Drucksteigerungen, Arch. Ges. Physiol. 274, 567-580 (1962).

12 K. Thurau, P. Deetjen and K. Kramer, Hämodynamik des Nierenmarks, Arch. Ges. Physiol. 270, 270-285 (1960).

13 J. M. Davis and J. Schnermann, The effect of antidiuretic hormone on the distribution of nephron filtration rates in rats with hereditary diabetes insipidus, Pflügers Arch. 330, 323-334 (1971).

14 J. Fourman and G. C. Kennedy, An effect of antidiuretic hormone on the flow of blood through the vasa recta of the rat kidney, J. Endocrin. 35, 173-177 (1966).

15 M. D. Johnson, C. S. Park and R. L. Malvin, Antidiuretic hormone and the distribution of renal cortical blood flow, Am. J. Physiol, in press. 